\title{
Application of an Inhomogeneous Stress (Patch) Model to Complex Subduction Zone Earthquakes: A Discrete Interaction Matrix Approach
}

\author{
JOHN B. RUNDLE \\ Sandia National Laboratories, Albuquerque, New Mexico \\ Hiroo KanAMORI \\ Seismological Laboratory, California Institute of Technology, Pasadena
}

\begin{abstract}
In recent years it has been recognized that the level of shear and normal stress along a fault can vary; thus the stress is spatially and temporally inhomogeneous. Moreover, it has also been suspected that faults might interact in some way, with the result that a variety of earthquake magnitudes might be produced along a given length of fault at varying times. In order to explore these ideas we have developed a quantitative formalism, which we call the interaction matrix method, to express the influence of one fault upon another. This matrix is calculated by use of the energy change for a system of interacting cracks or faults and therefore gives energy-consistent results. Specifically, the interaction matrix relates the area-averaged stress on the fault segment to the area-averaged slip state on all the other fault segments in the system. Since any fault can be subdivided into an arbitrary number of fault segments, the interaction matrix can have arbitrary dimension; in fact, the continuum limit is recovered as the dimension of the matrix approaches infinity. We combine this matrix method with a segmentation, or "patch," model for earthquakes, in which each discrete segment of a fault has the same coseismic stress change (defined as the difference between the driving stress at which healing occurs minus the driving stress at which sliding starts) each time it slips. We show that slip on a patch during an earthquake can vary substantially, depending on how it interacts with other nearby patches. In this model it is quite possible for the spatial distribution of stress on the fault following an event to be again in a spatially inhomogeneous state, rather than in a uniform state, as is often assumed. Hence the seismic moment produced by an earthquake on a given set of patches can vary substantially, depending on the sequence of sliding and healing on the different patches. To apply these ideas, we devised a means to calculate the interaction matrix elements and used them to quantitatively examine earthquake sequences off the Colombia-Ecuador coast and in the Nankai Trough near Japan.
\end{abstract}

\section{INTRODUCTION}

A great deal of attention has recently been focused on the earthquake mechanism and the extent to which an inhomogeneous stress distribution on faults controls the magnitude and recurrence properties of earthquakes (see, for example, Das and $\mathrm{Aki}$ [1977], Aki [1979], Madariaga [1979], Rudnicki and Kanamori [1981], Kanamori [1981], Lay et al. [1982], and Rudnicki et al. [1984]). This work has led to two proposals for the earthquake mechanism: that faulting nucleates in weak zones, with the extent controlled by the presence of strong "barriers," or that failure of a strong "asperity" drives faulting on surrounding weaker sections of the fault plane. In both cases it is assumed that alternating strong and weak sections occur along faults.

In both the asperity and the barrier model the concept of strong and weak regions on a fault plays a central role. In fact, the failure strength of a region ("patch") on a fault does not determine the slip or moment produced on that patch but only influences the initiation time of rupture relative to other nearby patches. Rather, it is the patch's coseismic stress change (or stress change for short, defined as the driving shear stress on the patch at the time sliding ends minus the driving shear stress on the patch at the time sliding begins) and the patch interaction effects which determine the patch moment

\section{Copyright 1987 by the American Geophysical Union.}

Paper number 6B6036. 0148-0227/87/006B-6036\$05.00 release or slip. In addition, by determining the amount of slip the stress change and the interaction effects also influence the recurrence time for slip on the patch. This will become clearer in the following discussions.

Consider Figure 1a, in which we represent two equal size patches (A and B). In this paper we use only simple, "old" frictional concepts, in which one assumes the existence of two frictional moduli: a static coefficient of friction $\mu_{s}$ and a dynamic coefficient of friction $\mu_{d}$. We assume that the level of shear stress on each patch generally rises with time, except during earthquakes, when slip on the patches lowers the stress. The value of shear stress at which sliding initiates on, say, patch $A$ we call the static strength $S_{A}$, and it is equal to $\left(\mu_{s}\right)_{\mathrm{A}} N_{\mathrm{A}}$, where $N_{\mathrm{A}}$ is equal in magnitude to the average compressional stress on the patch. As the shear stress gradually rises on the patch, during the time interval between earthquakes, no sliding occurs until the level of shear stress equals $S_{\mathrm{A}}$. This behavior is usually called Amonton's law [Jaeger and Cook, 1976]. Sliding thus initiates and continues until the shear stress level has declined to equal the dynamic strength $D_{\mathrm{A}}$; this quantity in turn is equal to $\left(\mu_{\mathrm{d}}\right)_{\mathrm{A}} N_{\mathrm{A}}$. At this point, slip on the patch terminates, healing occurs, and no further slip can take place until the level of shear stress has again risen back to $S_{\mathrm{A}}$ (note that the coseismic stress change $\sigma$ defined previously is therefore equal to $D_{\mathrm{A}}-S_{\mathrm{A}}$ ). These ideas therefore ignore much of the recent work on rate-dependent friction laws and slip weakening [Dieterich, 1981; Stuart, 1979; Ruina, 1983; Rice, 1983]. Moreover, it would probably be more accurate to consider $\left(\mu_{\mathrm{d}}\right)_{\mathrm{A}} N_{\mathrm{A}}$ to be an "arrest strength," as distinct 


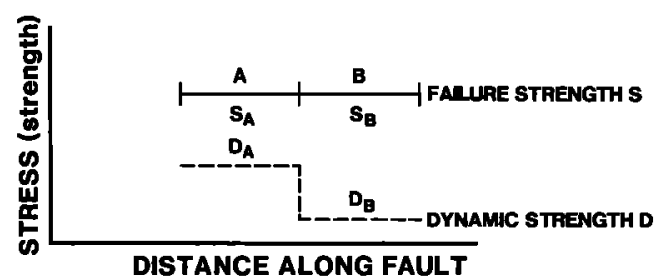

(a)

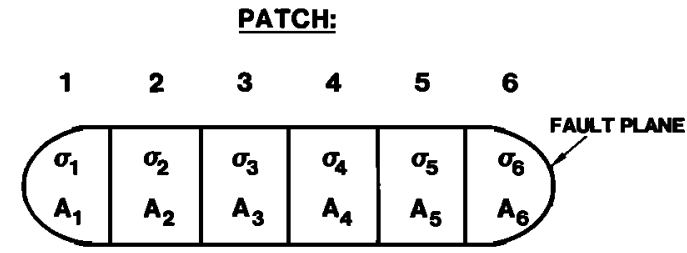

(b)

Fig. 1. (a) Schematic drawing of the dependence of failure strength with distance along a model fault. Patch $\mathrm{A}$ and patch $\mathrm{B}$ both have the same failure strength but have different dynamic strengths. (b) Schematic drawing of a planar fault consisting of six patches, the $i$ th patch having coseismic stress change $\sigma_{i}$ and area $A_{i}$.

from the "friction stress" or the "kinetic stress." Physically, one expects overshoot to occur during dynamic sliding, which will cause the fault patch to heal at a driving stress lower than the sliding friction. This should be the state at which the net stress (frictional plus elastic), having accelerated the surrounding fault blocks initially, has now decelerated them to zero velocity. For an isolated fault patch we might expect that $\mu_{d}=\mu_{f}-\left(\mu_{s}-\mu_{f}\right)$, where $\mu_{d}$ is the dynamic or "arrest" friction, $\mu_{f}$ is the sliding or "kinetic" friction, and $\mu_{s}$ is the static or "breaking" friction [see Rice and Tse, 1986]. We will assume that this "arrest strength" is roughly a constant for each fault patch and, for convenience, will continue to call it the dynamic strength.

This view of a fault plane is rather "macroscopic" in character. Obviously, each patch might also have "microstructure," giving rise to microearthquakes, on length scales considerably smaller than the patch length. Furthermore, these microevents might serve the purpose of homogenizing the stress field upon the patch, thereby allowing the patch to behave as a coherent unit. Slip in these events should be completely negligible compared to that in major events, however, if the notion of a patch is to have any meaning.

Returning to Figure $1 a$, we show two patches with the same static strengths but with different dynamic strengths. If the level of shear stress on both patches reaches the static strength at the same instant, both patches will begin sliding at the same time. Slip will continue on each patch until the shear stress falls to the dynamic strength, at which time healing will occur and slip will stop. If healing occurs at the same instant on both patches, the level of stress on each patch at the conclusion of sliding will be equal to the dynamic strength of each patch. In the case shown in Figure $1 a$, patch $\mathbf{B}$ will have slipped farther and will have contributed a larger moment release than patch $A$. If, however, slip ends and healing occurs on one of the patches before the other stops sliding, the final level of shear stress on the patch which stops first will be different from its dynamic strength. This is because the other patch, which is still sliding, continues to change the shear stress in its environs as long as it continues to slide. By contrast, the level of shear stress on the patch which stops last will be equal to its dynamic strength. At the conclusion of slip on both patches, static equilibrium should prevail. It is therefore the sequence of slip initiation and healing on all the patches, in addition to the stress change on each patch, which determines the final level of shear stress and moment release on each patch. In section 2 we will show that for a planar fault with patches, the case where slip initiation begins on all patches simultaneously, and subsequent healing occurs on all patches simultaneously, always produces the maximum possible moment release for the fault. Since on the average, slip on the fault keeps up with its average geologic rate, larger slip leads to a longer recurrence interval and vice versa. Hence by determining the size of slip in given event the detailed sequence of slip initiation and healing must also play a large role in determining the recurrence time.

Now consider Figure $2 b$. Here we show a hypothetical fault plane made up of a series of patches. Each has a characteristic coseismic stress change $\sigma_{i}$ and an associated patch area $\boldsymbol{A}_{\boldsymbol{i}}$. Now suppose that one of the stress changes, say $\sigma_{4}$, is much bigger in magnitude than any of the others:

$$
\begin{gathered}
\left|\sigma_{i}\right|=\sigma \quad i \neq 4 \\
\left|\sigma_{4}\right| \gg|\sigma|
\end{gathered}
$$

As we show in section 2, if patch 4 begins to slip, it tends to raise the level of stress on its neighbor patches. Since patch 4 has such a large coseismic stress change, it would tend to raise the level of stress in its neighbors by a proportionately large amount. Because the neighboring patches can only sustain relatively small stress increases (generally about equal to minus the coseismic stress change at most) without beginning to slip, they will, in fact, probably begin to slide. If patches 3 and 5 in turn trigger slip in their neighbors, the entire fault

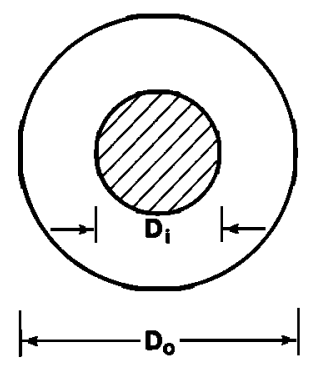

(a)

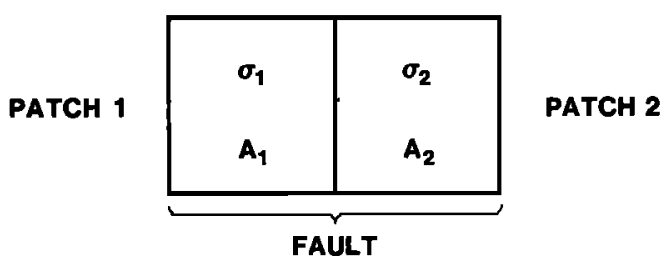

(b)

Fig. 2. (a) Inhomogeneous fault model. Inner diameter is $D_{i}$, and outer diameter is $D_{o^{-}}$Inner and outer regions can have different stress states (see Rundle et al. [1984] and equations (5)-(8)). (b) Schematic drawing of a planar fault consisting of two patches, each with stress change $\sigma_{i}$ and area $A_{i}$. 
plane may wind up sliding. In this case one might say that patch 4 is an asperity and that it controls sliding on the remainder of the fault plane through its ability to trigger slip on its neighbors.

Now suppose that the end patches, 1 and 6, have stress changes that are much larger in magnitude than any of the other four:

$$
\begin{gathered}
\left|\sigma_{1}\right|=\left|\sigma_{6}\right|=|\sigma| \\
|\sigma| \gg\left|\sigma_{\imath}\right| \quad i=2,3,4,5
\end{gathered}
$$

Suppose now that sliding initiates somewhere in the middle of the fault plane, say on patch 3 . As patch 3 slips, it may be able to raise the level of stress on its neighbors to the point where they in turn begin to slip, and sliding may thus progress along the fault plane until the end patches are encountered. Depending on the stress state in the end patches, the increase in stress needed to initiate sliding in the ends may be as high as minus their coseismic stress change, a very large amount. As we show in section 2, the amount of stress increase a patch can impart to its neighbors is proportional to minus its own coseismic stress change. If the patches are of equal size, the constant of proportionality is less than 1 . Hence under the scenario presented by (3) $(4)$, it is unikely that patches 2 and 5 could induce patches 1 and 6 to begin sliding. Slip would thus be confined to patches $2-5$. In this case, we might say that patches 1 and 6 were barriers and prevented sliding from progressing farther.

In the following, we quantitatively examine the implications of this kind of patch model for faults. We do not address the problem of why various pieces of the fault plane should have constant coseismic stress changes; perhaps these are the result of dynamic, inertia-controlled processes operating at the time of sliding (see, for example, Rice and Tse [1986]). Previous authors [Madariaga, 1979; Rudnicki and Kanamori, 1981; Rundle et al., 1984; Stuart, 1985] have used various subsets of these ideas without, evidently, exploring the complexity of moment release possible under various scenarios of slip initiation and healing. We pursue this line of inquiry in section 2 by introducing an interaction matrix approach and eventually apply these ideas to explaining the variation of moment release observed along the Colombia-Ecuador and Nankai troughs.

\section{INTERACTION MATRIX APPROACH}

We begin by considering the circular fault shown in Figure 2a. Both stress and slip are allowed to have independent values in the outer $(o)$ and inner (i) regions of the fault. Analytic expressions for the area-averaged offsets on the outer and inner regions, $\delta_{o}, \delta_{i}$, respectively, in terms of the area-averaged shear stress on the same regions, $\tau_{o}, \tau_{i}$, can be derived in the limit that the inner diameter is small compared to the outer diameter $\left(D_{i} / D_{o} \rightarrow 0\right)$ :

$$
\begin{gathered}
\delta_{o}=\frac{\tau^{\infty}-\tau_{o}}{2 \mu} \frac{D_{o}}{\eta}+\gamma \frac{\tau_{o}-\tau_{i}}{2 \mu} \frac{D_{i}}{\xi} \\
\delta_{i}=\frac{\tau^{\infty}-\tau_{o}}{2 \mu} \frac{D_{o}}{\kappa}+\frac{\tau_{o}-\tau_{t}}{2 \mu} \frac{D_{i}}{\xi}
\end{gathered}
$$

where

$$
\begin{gathered}
\xi=\frac{3 \pi(2-v)}{16(1-v)} \quad \eta=\frac{\xi}{\left(1-\left(D_{i} / D_{o}\right)^{2}\right)^{1 / 2}} \\
\kappa=\frac{\left(D_{i} / D_{o}\right)^{2} \xi}{\left[1-\left(1-\left(D_{\imath} / D_{o}\right)^{2}\right)^{3 / 2}\right]}
\end{gathered}
$$

and where $\gamma$ is a function of $\left(D_{i} / D_{o}\right)$ to be discussed below. In these equations, $\tau^{\infty}$ is a constant far-field shear stress, $\mu$ is the shear modulus, and $D_{o}, D_{i}$ are the outer and inner radii of the circular regions (see Figure 2). The quantities $\kappa, \eta$, and $\xi$ are constant functions of Poisson's ratio $v$ and are given below. Equations (5) differ slightly from the equations given by Rundle et al. [1984] by the appearance of $\eta$ and $\kappa$ in place of $\xi$. It was discovered in a reanalysis of the problem whose solution is given in (5) that the definition of "area average" by Rundle et al. [1984] was ambiguous. Equations (5) employ area averages over the inner and outer regions instead of only averages over the entire fault, as were used by Rundle et al. [1984]. We believe that equations (5) therefore represent a more satisfactory solution to the problem. It will be noted that $\kappa$ and $\eta$ deviate from $\xi$ by at most $33 \%$, for $D_{i} / D_{o} \leq 0.5$. The results quoted by Rundle et al. [1984] are only marginally affected. In particular, the specific numerical examples shown in Figures 7 and 9 of Rundle et al. [1984] are altered. In this case, wherein the outer region was considered to have a large coseismic stress change and the inner disk had a small coseismic stress change, no steady slip occurs within the inner disk during the time between major events.

Inverting equations (5), we find that

$$
\begin{aligned}
& \tau_{o}=A_{1} \delta_{o}+A_{2} \delta_{i}+\tau^{\infty} \\
& \tau_{i}=B_{1} \delta_{i}+B_{2} \delta_{o}+\tau^{\infty}
\end{aligned}
$$

Retaining only the first terms in $D_{i}$ and $D_{o}$, we find that

$$
\begin{gathered}
A_{1}=\frac{-2 \mu \xi}{D_{o}} \quad B_{1}=\frac{-2 \mu \xi}{D_{\imath}} \\
A_{2}=\frac{3 \mu \xi}{D_{o}}\left(D_{i} / D_{o}\right)
\end{gathered}
$$

Obviously,

$$
A_{1}, B_{1}<0 \quad A_{2}, B_{2}>0
$$

so that $A_{1}$ and $B_{1}$ are stress relief terms, whereas $A_{2}, B_{2}$ are stress increase terms. Alternatively, one can think of these terms as self-stress, and interaction stress terms, respectively.

Rundle et al. [1984] estimated the quantity $\gamma$ by an approximate technique and found it to be $\left(D_{v} / D_{o}\right)^{2}$ to leading order. It has since been found (see (14) below, as well as microfiche Appendix $\mathrm{A}^{1}$ ) that the ratio of the terms $A_{2} / B_{2}$ must equal the ratio of the areas, in this case $\left(D_{i} / D_{0}\right)^{2}$. This property comes from the definition of the "interaction matrix" (see Appendix A). Equation (14) is, in fact, an attractive result, since if it is assumed that the form of (5) is correct for small aspect ratios (with $\gamma$ unknown), then $\gamma$ can be determined as a function of $\left(D_{i} / D_{o}\right)$ by requiring property (14) to hold. Using this technique, we determined $\gamma$ to be

$$
\gamma \approx 1.5\left(D_{i} / D_{o}\right)-\left(D_{i} / D_{o}\right)^{2}+\cdots
$$

Note that the definitions of $A_{1}, A_{2}, B_{1}, B_{2}$ given below (7) are consistent with this definition of $\gamma$ to leading order and with (14). Again, using this value for $\gamma$ does not significantly change the numerical values or the qualitative behavior of the results given by Rundle et al. [1984].

The model described by (5)-(8) is a special case of a general

\footnotetext{
${ }^{1}$ Appendices A-D are available with entire article on microfiche. Order from American Geophysical Union, 2000 Florida Avenue, N.W., Washington, DC 20009. Document B87-007; \$2.50. Payment must accompany order.
} 
class of interacting two-patch models described by the equations (see Appendix A; see also Figure $2 b$ ):

$$
\begin{aligned}
& \tau_{1}=a_{11} \delta_{1}+a_{12} \delta_{2} \\
& \tau_{2}=a_{21} \delta_{1}+a_{22} \delta_{2}
\end{aligned}
$$

where

$$
\begin{aligned}
& a_{11}, a_{22}<0 \\
& a_{12}, a_{21}>0
\end{aligned}
$$

and where the determinant of coefficients $\Delta$ :

$$
\Delta=a_{11} a_{22}-a_{12} a_{21}>0
$$

More generally, if one has $N$ patches, (11) is replaced by (see Appendix C)

$$
\operatorname{sgn}\{\Delta\}=(-)^{N}
$$

It can be shown that (12) applies also to all the principal minors of the matrix $a_{i j}$, where in this case, $N$ is replaced by $M$, the dimension of the minor; these properties of the interaction matrix are proved in Appendix $\mathbf{C}$. While, in general, it may happen that the second of conditions (10) is violated (for example, this may happen for nonplanar faults), we confine our attention to planar faults for which (10)-(12) hold (equation (12) also holds for nonplanar faults; see Appendix C). Note that even if $(10 b)$ is violated, (11) or (12) must always hold. Equation (12) can be interpreted as a statement of the principle that slip on a patch affects the level of stress more on itself than on its surroundings. Equations (11) and (12) can be thus regarded as a condition of physical reality.

We call the matrix of quantities defined by $a_{i j}$ the elastic interaction matrix. Note that the $a_{i j}$ are calculated as integrals of Green's functions. In particular, these coefficients are area integrals of the (known) function $T_{k l}{ }^{m n}\left(x_{i}-x_{j}\right)$, which gives the average in-plane shear stress at location $x_{i}$ due to an isolated (single) element of fault at location $x_{j}$ [see Maruyama, 1964, equation 1.15]. Expressed mathematically, one has (see Appendix A)

$$
\begin{gathered}
a_{i j}=\frac{1}{A_{i}}\left(\frac{1}{2}\right)\left[b_{i j}+b_{j l}\right] \\
b_{i j}=\int_{A_{i}} \int_{A_{j}} U^{i}\left(x_{i}\right) U^{j}\left(x_{j}\right) v_{k}{ }^{i} v_{m}{ }^{j} T_{k l} m n\left(x_{i}-x_{j}\right) \eta_{l}{ }^{i} \eta_{n}^{j} d A_{j} d A_{i}
\end{gathered}
$$

(no sum on $i, j$; sum on $k, l, m, n$ ), where $b_{j i}$ is obtained from $b_{i j}$ by interchanging the indices $i$ and $j ; A_{i}$ is the area of the $i$ th patch; $v_{k}{ }^{i}, v_{m}{ }^{j}$, are the slip unit vectors on the $i$ th and $j$ th patches; and $\eta_{l}{ }^{i}, \eta_{n}{ }^{j}$, are the unit normals for the $i$ th and $j$ th patches, respectively. Note that $U^{i}\left(x_{i}\right), U^{j}\left(x_{j}\right)$, are (unknown) offsets across patches $i$ and $j$, whose area averages over patches $i$ and $j$ are equal to unity (see Appendix A for a more complete discussion). We assume that sufficiently smooth offsets can be found which correspond to uniform stress change on each patch. Note also that the $b_{i j}$ in (13) defines energy integrals (see Appendices A and D): One has a force due to slip on the $j$ th patch acting through a displacement on the $i$ th patch and vice versa. For the diagonal elements $a_{i i}$, note that one of the integrations must be a Cauchy principal value integral. The definition of the quantities $a_{i j}$ therefore ensures that the stress $\tau_{i}$ and the offset $\delta_{i}$ on the $i$ th patch are work conjugate quantities.

Since the coefficients $a_{i j}$ contain the quantities $U^{n}\left(x_{n}\right)$ in the integrands, the $a_{i j}$ appear to contain information on the slip and stress state of the medium. One might think that it would then be necessary to calculate the coeflicients for each different stress state of the cracks. However, it can be shown (see Appendix A) by the use of superposition of stress and the uniqueness theorem that the $a_{i j}$ are entirely independent of the stress state of the medium and therefore also of the slip state on the cracks. Thus the $a_{i j}$ are functions only of the geometry of the cracks (and, of course, the direction of the resolved, in-plane slip, and shear traction).

A significant property of the off-diagonal elements of the matrix $a_{i j}(i \neq j)$ is that

$$
A_{i} a_{i j}=A_{j} a_{j i}
$$

(no sum on $i, j$ ). This property is clearly evident from the definition (13). In general, these stress exchange or stress interaction coefficients are not easy to compute by exact methods. In Appendix B (on microfiche) we discuss approximate, but sufficiently accurate, numerical methods of calculating matrix elements for rectangular areas. These methods involve the use of smoothed rectangular uniform dislocations: Some sort of smoothing is, of course, necessary since uniform dislocations have nonintegrable stress singularities at their edges. By contrast, faults can have no such singularities.

We now consider several problems of the kind mentioned in the introduction, where a variety of slip initiation and healing sequences can occur. Let $\sigma_{1}$ and $\sigma_{2}$ denote the coseismic stress change for patches 1 and 2 . Now consider the problem defined by the following sequence of events:

1. The level of shear stress rises in such a way that it meets the static strength $S_{i}$ of both patches 1 and 2 at the same instant.

2. Both patches begin to slide at exactly the same time.

3. The level of shear stress on each patch declines during the sliding event by each coseismic stress change $\sigma_{i}$, reaching the dynamic strength $D_{i}$ at the same instant.

4. Both patches heal at the same time.

We call this problem I, the case of simultaneous slip. We have drawn this problem graphically (in an obvious similarity to Feynman diagrams) in Figure $3 a$, in stress-time space. The stress release process on each patch is represented by a solidarrowed line, with the arrow indicating the direction of time increase. The two wavy lines connecting the arrowed lines indicate that the two patches communicate their respective states (starting at $t_{1}$ and stopping at $t_{2}$ ) to each other during the slip process via seismic waves. The dashed legs on the diagrams indicate change in stress when no sliding is occurring; thus this is a stress accumulation process. One interpretation of our model therefore explicitly assumes that the entire slip initiation sliding-healing process takes place on a time scale which is slower than the time it takes for seismic waves to propagate between the two patches. There is some limited evidence in support of this idea that the total slip (seismic plus aseismic) occurs on a time scale substantially longer than, say, the source dimension divided by the shear wave velocity [Kanamori and Stewart, 1979; Gladwin and Johnston, 1986]. This time scale for total moment release appears to be in the range of at least several hours. Another possible interpretation of our model is that it is an aggregate representation of a more complex dynamic process. In this view we assume that in addition to a "rupture front" which propagates outward from the hypocenter at some rupture velocity, there follows a "healing front" which (possibly) propagates outward from the hypocenter following the rupture 


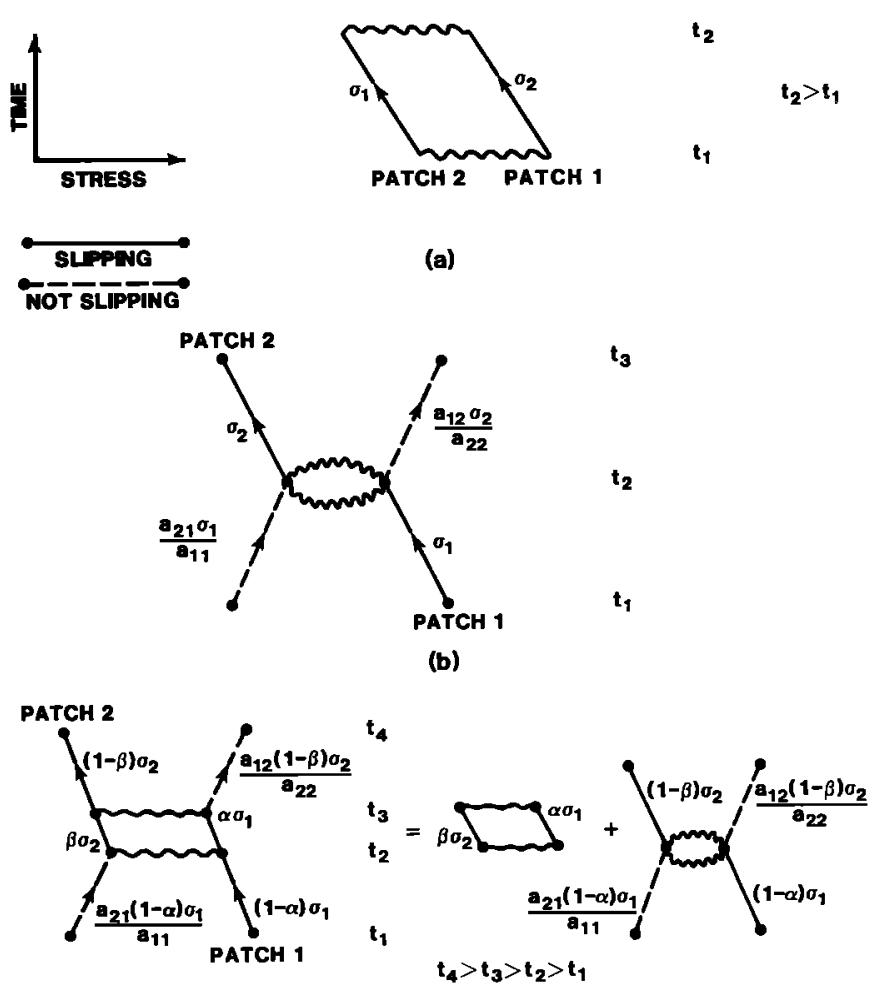

(c)

Fig. 3. Process diagrams in stress-time space (modeled after Feynman diagrams) for all possible modes of slip initiation and healing for the two patches shown in Figure $2 b$. The arrowed lines represent the trajectories of the two patches, and the wavy lines represent communication between the patches that occurs via seismic waves. $(a)$ Simultaneous slip (problem I). (b) Sequential shp (problem II). (c) An intermediate case (problem III). Figure $3 c$ also indicates that problem III can be decomposed into a problem I (but with different stress change) and a problem II (again with a different stress change).

front. Thus sliding and healing can occur on various pieces of the fault plane at the same time. Our simple model is then a simple representation of this much more complex process.

The solution to problem I is therefore given by the following set of equations:

$$
\begin{aligned}
& \sigma_{1}=a_{11} \delta_{1}{ }^{I}+a_{12} \delta_{2}{ }^{I} \\
& \sigma_{2}=a_{21} \delta_{1}{ }^{I}+a_{22} \delta_{2}^{I}
\end{aligned}
$$

where $\delta_{i}{ }^{1}$ is the slip for problem $I$ on the $i$ th patch. The solution to problem $I$ is easily found to be

$$
\begin{aligned}
& \delta_{1}{ }^{I}=\frac{\sigma_{1} a_{22}-\sigma_{2} a_{12}}{\Delta} \\
& \delta_{2}{ }^{I}=\frac{\sigma_{2} a_{11}-\sigma_{1} a_{21}}{\Delta}
\end{aligned}
$$

Now consider problem II, illustrated graphically by Figure $3 b$. In this problem we assume the following sequence of events, which occur all within the same earthquake:

1. The level of shear stress is such that it equals the static strength $S_{1}$ on patch 1 but is less than the static strength $S_{2}$ on patch 2 . In fact, we assume that the level of shear stress on patch 2 is $S_{2}-\left(a_{21} \sigma_{1} / a_{11}\right)$.

2. Patch 1 begins to slip at time $t_{1}$ and continues to slip until time $t_{2}$, when it heals.
3. While patch 1 is sliding, it is raising the level of stress on patch 2. This continues until time $t_{2}$, and just as patch 1 begins to heal, patch 2 begins to slip.

4. Patch 1 heals, and patch 2 continues to slip until time $t_{3}$, when it heals. During this process, sliding on patch 2 raises the level of stress on patch 1 to $D_{2}+\left(a_{12} \sigma_{2} / a_{22}\right)$.

This sequence of events is described by the system of equations

$$
\begin{aligned}
& \sigma_{1}=a_{11} \delta_{1}^{\mathrm{II}} \\
& \sigma_{2}=a_{22} \delta_{2}^{\mathrm{II}}
\end{aligned}
$$

with the trivial solutions

$$
\delta_{1}{ }^{\mathrm{II}}=\sigma_{1} / a_{11} \quad \delta_{2}{ }^{\mathrm{II}}=\sigma_{2} / a_{22}
$$

Now $\delta_{1}{ }^{\mathrm{I}}$ can be written

$$
\delta_{1}{ }^{I}=\frac{\sigma_{1}}{a_{11} \Delta^{\prime}}-\frac{\sigma_{2} a_{12}}{\Delta}
$$

where

$$
\Delta^{\prime}=1-\frac{a_{12} a_{12}}{a_{11} a_{22}}>0 \quad 1 \geq \Delta^{\prime}>0
$$

Recalling (10) and (11) and that $\sigma_{1}, \sigma_{2}<0$ by definition, we can rewrite (19) as

$$
\delta_{1}{ }^{\mathrm{I}}=\delta_{1}{ }^{\mathrm{II}} \cdot\{\text { factor greater than } 1\}+\{\text { positive number }\}
$$

leading to

$$
\delta_{1}{ }^{\mathrm{I}} \geq \delta_{1}{ }^{\mathrm{II}}
$$

In a similar way we can show that

$$
\delta_{2}{ }^{\mathrm{I}} \geq \delta_{2}^{\mathrm{II}}
$$

Defining the total seismic moment $M_{T}$ for $N$ patches as

$$
M_{T}=\mu \sum_{t=1}^{N} \delta_{t} A_{i}
$$

we have

$$
M_{T}^{\mathrm{I}} \geq M_{T}^{\mathrm{II}}
$$

Now consider problem III, illustrated graphically in Figure $3 c$. As shown in Figure $3 c$, the algebra for this process is reflected in a "pictorial algebra" of the graphs: The process can be written as the sum of two subprocesses. Figure 4 illustrates the same process but plotted in stress 1/stress 2 space (the ordinate is the shear stress on patch 1 , the abscissa is the shear stress on patch 2). Note that the system point stays within the same small volume bounded by $S_{1}, D_{1}$, and $S_{2}, D_{2}$. A third method of illustrating the process would be to plot it in stress displacement phase space. In that case, the system point would also occupy a closed volume. In problem III we have the following sequence of events:

1. The initial stress on patch 1 at time $t_{1}$ is $S_{1}$, while on patch 2 the initial stress is $S_{2}-\left(a_{21} / a_{11}\right)(1-\alpha) \sigma_{1}$, with $1 \geq \alpha \geq 0$.

2. Patch 1 begins sliding, decreasing its stress by an amount $(1-\alpha) \sigma_{1}$ at time $t_{2}$ and raising the stress on patch 2 by an amount $\left(a_{21} / a_{11}\right)(1-\alpha) \sigma_{1}$.

3. At time $t_{2}$, patch 2 begins to slide, and from $t_{2}$ to $t_{3}$, both patches slide together. During this time, patch 1 decreases its stress by $\alpha \sigma_{1}$, and patch 2 decreases its stress by $\beta \sigma_{2}, 1 \geq \beta \leq 0$. 
(a)

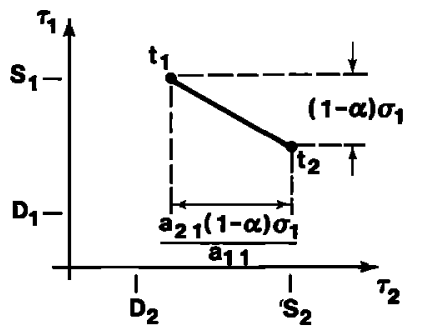

(b)

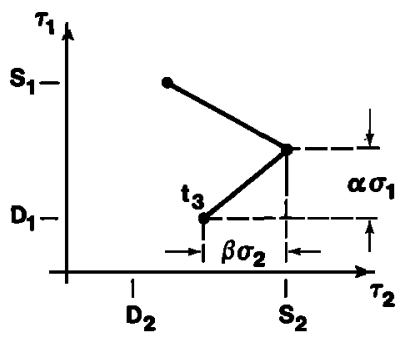

(c)

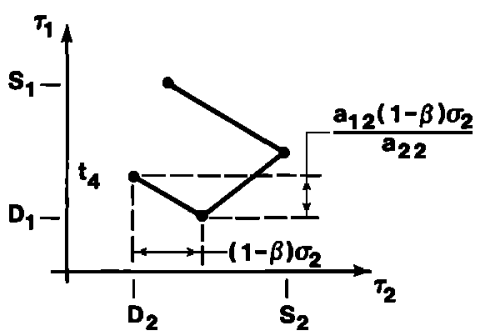

Fig. 4. Motion of the system point for the two patches of Figure $2 b$ in stress $1 /$ stress 2 space. The abscissa is for patch 2 , the ordinate for patch 1 , and static $S_{i}$ and dynamic $D_{i}$ stress states are indicated. Figures $4 a-4 c$ are for the same situation as illustrated in Figure $3 c$.

4. At time $t_{3}$, patch 1 heals, while patch 2 continues sliding, until at time $t_{4}$ it, too, heals. During this time the stress on patch 2 declines by $(1-\beta) \sigma_{2}$.

5. The final stress state on patch 2 is $D_{2}$ and on patch 1 is $D_{1}+\left(a_{12} / a_{22}\right)(1-\beta) \sigma_{2}$. This process is described by

$$
\begin{gathered}
(1-\alpha) \sigma_{1}=\delta_{1}^{(1)} \quad(1-\beta) \sigma_{2}=\delta_{2}{ }^{(1)} \\
\alpha \sigma_{1}=a_{11} \delta_{1}{ }^{(2)}+a_{12} \delta_{2}^{(2)} \\
\beta \sigma_{2}=a_{21} \delta_{1}^{(2)}+a_{22} \delta_{2}^{(2)}
\end{gathered}
$$

where

$$
\delta_{i}{ }^{\mathrm{III}}=\delta_{i}^{(1)}+\delta_{i}{ }^{(2)} \quad i=1,2
$$

The solution $\delta_{1}{ }^{\text {III }}$ to this problem is easily found to be

$$
\delta_{1}{ }^{\mathrm{III}}=\left[\frac{\alpha}{\Delta^{\prime}}+(1-\alpha)\right] \frac{\sigma_{1}}{a_{11}}-\frac{\beta a_{12} \sigma_{2}}{\Delta}
$$

Note that $\delta_{2}{ }^{\text {III }}$ can be easily obtained from $\delta_{1}{ }^{\text {III }}$ by interchange of $(\alpha, \beta)$ and the subscripts 1 and 2. Since (24) indicates that $\delta_{1}^{\text {III }}$ is a linear function of $(\alpha, \beta)$, we can evaluate the bounds for $\delta_{1}{ }^{\text {III }}$ by evaluating (24) at the four limit points $(\alpha, \beta):(0,0)$, $(0,1),(1,0),(1,1)$. Thus

$$
\begin{aligned}
& (0,0): \quad \delta_{1}{ }^{\mathrm{II}}=\delta_{1}{ }^{\mathrm{II}} \\
& (0,1): \quad \delta_{1}{ }^{\mathrm{II}}=\frac{\delta_{1}{ }^{\mathrm{II}}}{\Delta^{\prime}}
\end{aligned}
$$

hence $\delta_{1}{ }^{\text {II }} \leq \delta_{1}^{\text {III }} \leq \delta_{1}^{\text {I }}$

$$
(1,0) ; \quad \delta_{1}{ }^{\mathrm{II}}=\delta_{1}{ }^{\mathrm{II}}-\frac{a_{12} \sigma_{2}}{\Delta}
$$

hence $\delta_{1}{ }^{\text {II }} \leq \delta_{1}{ }^{\text {III }} \leq \delta_{1}{ }^{\mathrm{I}}$

$$
(1,1): \quad \delta_{1}{ }^{\mathrm{III}}=\delta_{1}{ }^{\mathrm{I}}
$$

Thus we have the basic result

$$
\delta_{i}^{\mathrm{II}} \leq \delta_{i}^{\mathrm{III}} \leq \delta_{t}^{\mathrm{I}} \quad i=1,2
$$

Using the definition (19) for total moment $M_{T}$, we also have the result

$$
M_{T}^{\text {II }} \leq M_{T}^{\text {III }} \leq M_{T}{ }^{I}
$$

Thus the largest possible total moment occurs when both patches begin sliding at the same time and heal at the same time. We call this the case of simultaneous slip. The smallest possible moment occurs when one patch completes its slip and heals immediately before the other begins sliding. We call this the case of sequential slip. Any sequence of sliding and healing during the same event produces a total moment which lies somewhere between these two limits.

\section{ApPlication to Colombia-Ecuador EARTHQUAKES}

As discussed by Kelleher [1972], Kanamori and McNally [1982], and Beck and Ruff [1984], the Colombia-Ecuador earthquake sequence consisted of a series of major events in 1906, 1942, 1958, and 1979 (see Figure 5). The latter three events ruptured essentially the same portion of the plate boundary (Figure 5) as the 1906 event evidently did. Magnitudes $M_{w}$ and moments $M_{0}$ for these events are given in Table 1. The moment for the 1906 event is, of course, somewhat uncertain but is based upon a combination of tsunami height relative to the much better recorded 1979 event, limited instrumental data, and local Rossi-Forel intensity observations. The moment for the 1942 event is based upon the size of the aftershock area and its general similarity to the 1958 event. The moment for the 1958 event is reliably derived from a Press-Ewing seismograph at Pasadena which also recorded the 1979 event. Assuming the same focal mechanism allows a determination of moment from the aftershock area and the Press-Ewing amplitude ratio. Moment for the 1979 event was determined from standard instrumental observations.

Accounting for the inevitable uncertainty in moment determination of the various events, Kanamori and McNally [1982] found that the ratio of moment of the 1906 event relative to the sum of moments for the later three events can reasonably range from about 2.7 to 7 . Of course, slip in 1906 may have occurred over a substantially larger area. However, we will show that the patch model can account for most of the difference in moment without requiring a larger fault area.

Using the results of section 2 , we can proceed to demonstrate that the patch model, including the concept of triggered slip, can account for the larger amount of slip when the patches slipped together in 1906. Consider the model shown in Figure 6 and given in Table 2. The 1906 fault plane is represented by the five patches shown in plan view in Figure 6, the geometry of which was taken from Beck and Ruff [1984]. Patches 1-3, composing a distance along strike of some 240 $\mathrm{km}$ and a width downdip of $100 \mathrm{~km}$, broke again during the 1979 event. Patches 4 and 5, representing the 1958 and 1942 events, respectively, are each $50 \mathrm{~km}$ along strike and $50 \mathrm{~km}$ downdip. The model values for static stress drop, both strike slip and dip slip, are given in Table 3 and are discussed below.

In order to solve the problem posed by the detailed geometry in Figure 6 it was necessary to go substantially beyond the 


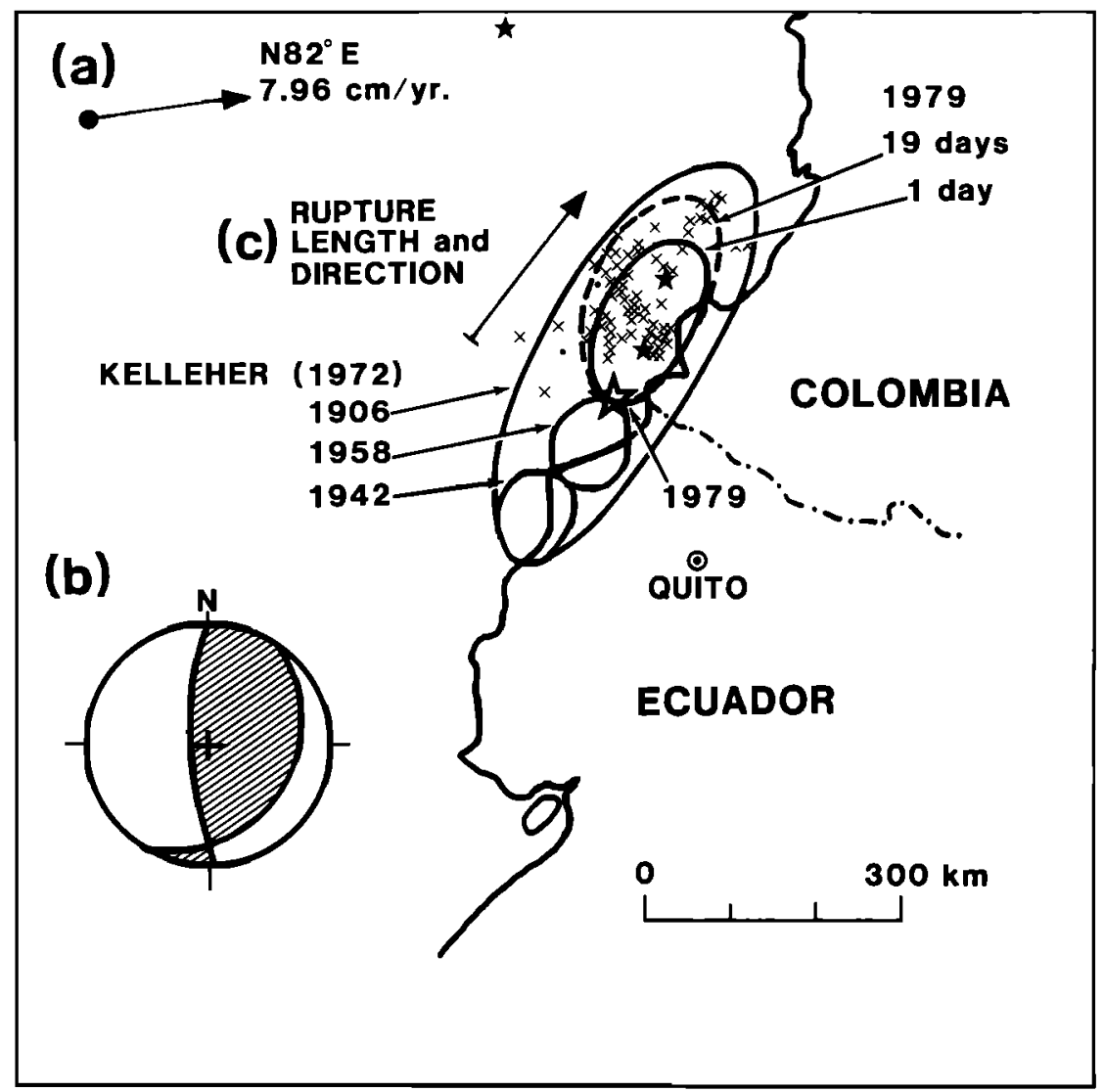

Fig. 5. Map of the Colombia earthquakes. (a) The plate convergence rate. (b) The lower half of the focal sphere for the 1979 event. (c) rupture length and direction. See Kanamori and McNally [1982] for details.

calculations discussed in section 2 using (5) $-(7)$. As discussed in detail in Appendix B (on microfiche), one needs to calculate the area-averaged stress changes on the $j$ th rectangular fault due to virtual slip on the $i$ th rectangular fault. Note that these stress changes have no singularities at the edges of the faults because obviously, the earth cannot support an infinite stress. These expressions are not available in the literature, so a means must be found to calculate them from dislocation solutions (which do have edge singularities) and which are readily available. This means that an edge-smoothing algorithm must be developed which will allow the uniform dislocation solutions to be "turned into" crack, or fault solutions. We devised such a procedure, and a detailed discussion is given in Appen$\operatorname{dix}$ B. We used the expressions for displacements from dipping strike-slip and dip-slip faults in an elastic half-space given by Mansinha and Smylie [1971], together with our smoothing algorithm, to derive expressions for the average in-plane shear stress for both the downdip and along-strike directions, on

TABLE 1. Moment Estimates for Colombia Earthquake Sequence

\begin{tabular}{ccc}
\hline Event & $M_{w}$ & $\begin{array}{c}M_{0}, \\
\times 10^{27} \mathrm{dyn} \mathrm{cm}\end{array}$ \\
\hline 1906 & 8.8 & $80-200$ \\
1942 & 7.6 & 3 \\
1958 & 7.7 & 5 \\
1979 & 8.2 & $25-35$ \\
\hline
\end{tabular}

Moments and magnitudes for the Colombia earthquake sequence [from Kanamori and McNally, 1982; Beck and Ruff, 1984]. arbitrary dipping faults within the half-space. Since both slip and frictional resistance are in the same direction as the shear traction on the fault surface, it can be shown that the analogous equations to (18)-(19) for $N$ patches are

$$
\begin{array}{r}
\sigma_{i}=\sum_{j=i}^{N}\left(a_{i j}{ }^{s} \cos ^{2} \alpha+a_{i j}{ }^{d} \sin ^{2} \alpha\right) \delta_{j}{ }^{T} \\
i=1, \cdots, N
\end{array}
$$

where $\alpha$ is the angle of slip $\left(0^{\circ}=\right.$ pure strike slip, and $90^{\circ}=$ pure thrust), $\sigma_{i}$ is the constant stress drop for the $j$ th patch, and $\delta_{j}{ }^{T}$ is the slip for the $j$ th patch in the direction specified by $\alpha$. Note that (28) is a consequence of the tensor transformation law and the fact that the strike-slip offset $\delta_{s}$ is related to the total offset $\delta^{T}$ by $\delta_{s}=\delta^{T} \cos \alpha$, etc. The quantities $a_{i j}{ }^{s}$ and $a_{i j}{ }^{d}$ are the stress interaction coefficients for the

\begin{tabular}{|c|c|c|c|c|}
\hline $\begin{array}{l}1906 \\
1979\end{array}$ & $\begin{array}{l}1906 \\
1979\end{array}$ & $\begin{array}{l}1906 \\
1979\end{array}$ & $\begin{array}{c}1906 \\
1958 \\
\text { (4) }\end{array}$ & $\begin{array}{c}1906 \\
1942 \\
\text { (5) }\end{array}$ \\
\hline (1) & (2) & (3) & & 10 \\
\hline
\end{tabular}

PATCHES: COLOMBIA - ECUADOR

Fig. 6. Patch model for the Colombia-Ecuador earthquakes. Geometric parameters for the patches (length, width, dip, slip angle) are given in Table 2. Patches 1-3 broke in the 1906 event and again in 1979. Patches 4 and 5 both broke in 1906, and in the 1958 and 1942 events, respectively. 
TABLE 2. Parameters for Colombia-Ecuador Earthquakes

\begin{tabular}{lccccc}
\hline & \multicolumn{5}{c}{ Patches } \\
\cline { 2 - 6 } & 1 & 2 & 3 & 4 & 5 \\
\hline Length, km & 120 & 75 & 45 & 50 & 50 \\
Width, km & 100 & 100 & 100 & 50 & 50 \\
Dip angle & $20^{\circ}$ & $20^{\circ}$ & $20^{\circ}$ & $20^{\circ}$ & $20^{\circ}$ \\
Slip angle* & $45^{\circ}$ & $45^{\circ}$ & $45^{\circ}$ & $45^{\circ}$ & $45^{\circ}$ \\
\hline
\end{tabular}

Faulting parameters for the Colombia-Ecuador earthquake fault patches. Refer to Figure 6 for details.

${ }^{*} 90^{\circ}=$ pure thrust.

$i$-jth patch for strike-slip and dip-slip motion, respectively. In these calculations it was assumed that shear stress in the downdip direction was due only to dip-slip motion and that shear stress in the along-strike direction was due only to strike-slip motion. In reality, dip-slip motion contributes a negligible amount to the shear stress in the along-strike direction and vice versa.

As part of a systematic examination of the predictions of the model computations, it was found that both strike-slip and dip-slip motion are reasonably efficient at increasing the stress level on neighboring patches, thus tending to trigger further slip on these neighbors. For the particular configuration shown in Figure 6 it was also found that the strike-slip motion was about $50 \%$ more efficient than the dip-slip motion in raising the level of stress on neighboring patches. For faulting in a purely elastic medium the patch-patch interaction is basically an edge effect, whose horizontal distance scale is set by the minimum dimension of the fault plane. Hence if the various patches are similar in size, the patch-patch interaction for an elastic medium is basically a nearest-neighbor interaction.

In order to model the $1906,1942,1958$, and 1979 earthquakes we made the basic assumption that the 1906 event represented the simultaneous slip of all five of the patches shown in Figure 6 and was therefore the maximum moment event of which the five patches were capable. Patch parameters for these five patches are given in Table 2. We assume that the 1942 and 1958 events represent slip of only one patch in each event, patches 5 and 4 , respectively. In addition, we hypothesize that the 1979 event represents the totally sequential slip of patches 3, 2, and 1. Hence we assume that the 1979 event was the minimum size event for the three patches. Thus we hope to account for the difference in size of the 1906 event compared to the later events solely by variations in the sequence of slip initiation and healing on the various patches.

TABLE 3. Summary of Calculated Results for Colombia-Ecuador Earthquake Model

\begin{tabular}{cccc}
\hline Patch & $\begin{array}{c}\text { Patch Stress Drop, } \\
\text { MPa }\end{array}$ & $\begin{array}{c}\text { Single Patch Slip, } \\
\text { m }\end{array}$ & $\begin{array}{c}\text { 1906 Slip, } \\
\text { m }\end{array}$ \\
\hline 1 & -.740 & 1.0 & 5.8 \\
2 & -6.286 & 6.0 & 11.8 \\
3 & -3.283 & 2.0 & 8.0 \\
4 & -3.349 & 2.0 & 6.9 \\
5 & -3.349 & 2.0 & 4.5 \\
\hline
\end{tabular}

Patch stress drops were calculated by finding the stress drop for each patch, slipping alone, that give the slip values shown in column 3 [from Beck and Ruff, 1984]. The slip calculated for all five patches slipping simultaneously is given in column 4.
Since Beck and Ruff [1984] give the moment for each of the patches $1-4$ in 1958 and 1979 , the static stress drop for each patch can be found in a straightforward way. Because we have assumed that the later events represent minimum size events, for which we know the moment on the various patches, and therefore the slip, we can thus calculate the stress change. For each patch we calculate the offset for a unit stress change and then find the proper stress change by dividing the observed slip by the calculated offset. We assume that the static stress drop for patch 5 is the same as that for patch 4 due to the similarity in size and estimated moment. We also make the assumption that the ratio of strike slip to dip slip (the slip angle) for the events is the same as the angle of convergence relative to the strike of the fault zone, essentially $45^{\circ}$. This assumption is supported by the focal mechanism for the 1979 event [Kanamori and McNally, 1982].

Thus the focal mechanism for the 1906 event is calculated by simply assuming that all five patches slip simultaneously, as discussed in section 2. The result of the calculation is shown in Table 3 for each of the five patches in Figure 6. The enhanced effect on the slip of each patch due to the simultaneous slip of all five patches is obvious. The total moment calculated for the 1906 event is then $133 \times 10^{27} \mathrm{dyn} \mathrm{cm}$, within the range found by Kanamori and McNally [1982].

\section{Application to the Nankai Trough EARTHQUAKES}

Historical records of the events occurring in the Nankai Trough off southwest Japan indicate great earthquakes (Figure 7) dating back to at least 684 A.D. [Ando, 1975]. The latest events in the series are the Tonankai (1944) and Nankaido (1946) earthquakes, which both had seismic moments of about $1.8 \times 10^{28}$ dyn cm determined from long-period surface waves [Kanamori, 1972]. The geodetically inferred moments for the two events are somewhat larger, about $2.2 \times 10^{28}$ dyn $\mathrm{cm}$ for the Tonankai event and $7.9 \times 10^{28} \mathrm{dyn} \mathrm{cm}$ for the Nankaido earthquake. Ando's [1975] model for the five most recent events is shown schematically in Figure 8; moments and geometric parameters for these five events are given in Table 4. Evidently, there exist four fault planes in the Nankai Trough which are responsible for the continuing series of earthquakes there. Considerable instrumental, geodetic, intensity, and tide gauge data exist for the two most recent events, the 1946 Nankaido and 1944 Tonankai earthquakes, as discussed by Kanamori [1972]. Quantitative data on the 1854 Ansei earthquakes and on the 1707 Hoei event originate from historic tidal records and intensity descriptions. While there is inevitable uncertainty about the correct fault plane models for these earlier events, the similarity of the deformation and damage patterns suggests that these earlier events occurred on essentially the same fault planes as the 1944 and 1946 events. Additionally, the Ansei I event, which occurred only about 32 hours earlier than the Ansei II event, clearly did considerably more damage to the Tokai district farther east [Ando, 1975], hence the addition of fault plane $D$. It is also clear that the 1707 Hoei earthquake must have been considerably larger than any of the other historical events in the Nankai Trough. Moreover, the pattern of damage, coastal uplift, and shaking intensity indicates that the Hoei event probably involved all four fault planes and that the offsets on them must have been considerably larger than those for the later events. These conclusions are summarized in Table 4 and in Figure 8.

The problem, then, is to explain why the slip in the 1707 Hoei earthquake was so much larger than the slip in any of 


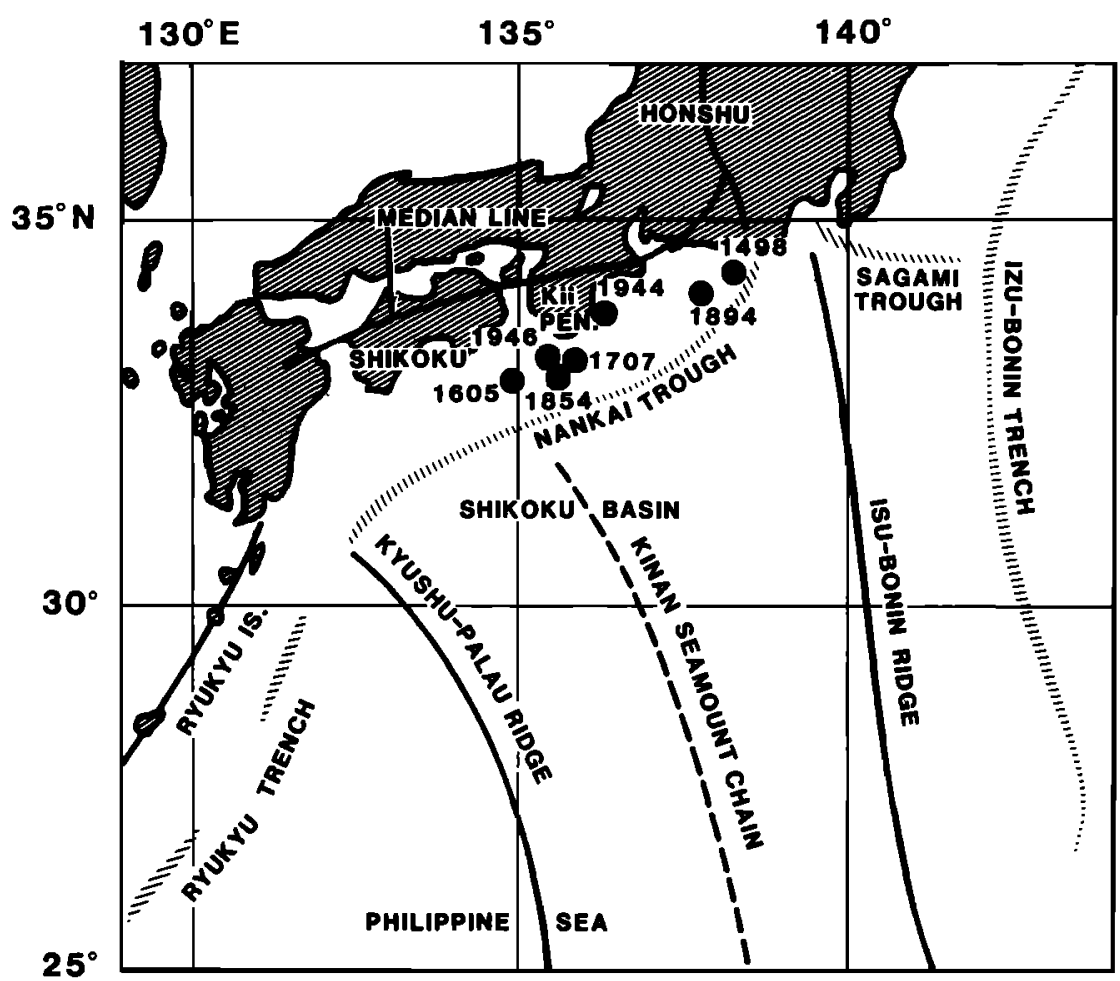

Fig. 7. Location map for events in the Nankai Trough off the coast of Japan (adapted from Ando [1975]). Approximate epicenters for great earthquakes in the trough are shown as solid circles next to the date of occurrence.

the later events. Once again, we hypothesize that the four fault planes in Ando's [1975] model represent patches with constant stress change and area during each of the events in the trough. These patches are shown in Figure $9 a$, and the patch parame-

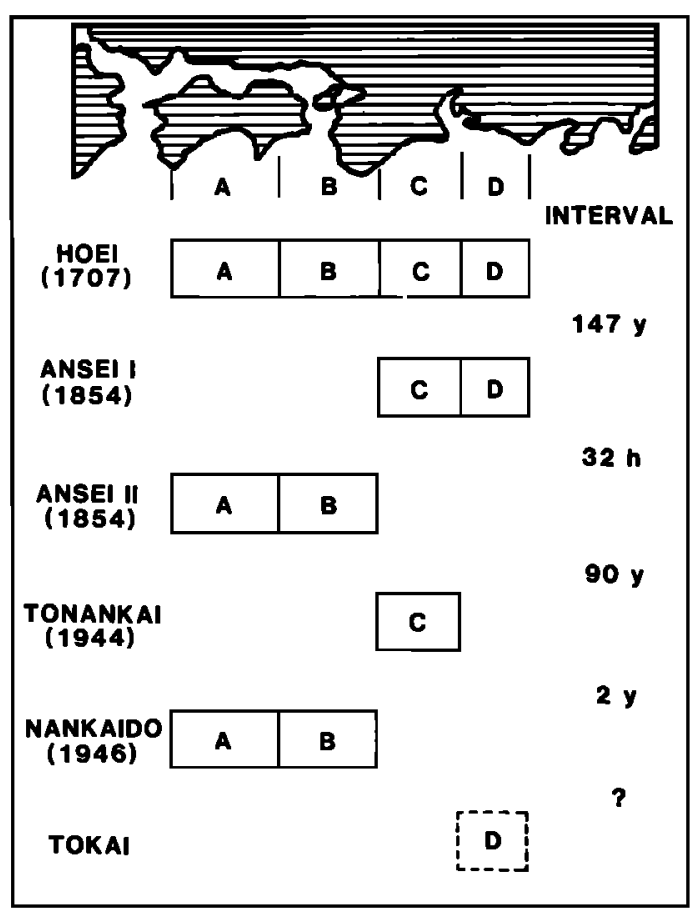

Fig. 8. Schematic representation of the latest five events in the Nankai Trough sequence. The cyclic nature of the events is suggested, as well as the conceptual model of events occurring on the same four fault planes. The dashed square indicates the hypothesized Tokai earthquake to come ters are given in Table 5. The model is basically the same model as given by Ando [1975, Table 1]. One difference is that although Ando [1975] shows a strike azimuth for patches 1 and 2 (Ando's A and B) that differs from the strike azimuth for 3 and 4 (Ando's $C$ and D) by $10^{\circ}$, our patches in Figure $9 a$ have all the same strike angle and are contiguous. Moreover, we assume that all of the patches have an identical dip angle of $25^{\circ}$, whereas Ando [1975] uses a dip angle of $20^{\circ}$ for patch 1 (A) and dip angles of $25^{\circ}$ for patches $2-4$ (B-D). Finally, we assume a uniform slip angle of $68^{\circ}$, instead of $63.4^{\circ}$ for patches 1 and 2 ( $A$ and $B$ ) and $71.6^{\circ}$ for patches 3 and 4 (C and D). These changes, made for the sake of simplicity, affect the results only negligibly. In all other respects the fault planes in Figure $9 a$ are identical to those of Ando [1975], and details may be found there.

To calculate the values for the patch offsets, we assumed that the 1707 earthquake represents the totally simultaneous slip of all four patches and that the other four events in Table 4 represent totally sequential slip of the various patches involved in each event. Thus the later events can be used to find

TABLE 4. Recent Nankai Trough Earthquakes

\begin{tabular}{lcccccc}
\hline & & \multicolumn{4}{c}{ Patch Slip, m } \\
\cline { 3 - 7 } \multicolumn{1}{c}{ Event } & $\begin{array}{c}\text { Geodetic Moment, } \\
\times 10^{27} \text { dyn cm }\end{array}$ & 1(A) & 2(B) & $3(\mathrm{C})$ & 4(D) \\
\hline Nankaido (1946) & 79 & 6 & 4 & $\ldots$ & $\ldots$ \\
Tonankai (1944) & 22 & $\ldots$ & $\ldots$ & 4 & $\ldots$ \\
Ansei II (1854) & 79 & 6 & 4 & $\ldots$ & $\ldots$ \\
Ansei I (1854) & 39 & $\ldots$ & $\ldots$ & 4 & 4 \\
Hoei (1707) & 240 & 12 & 8 & 8 & 8 \\
\hline
\end{tabular}

Summary of slip data for the five most recent great earthquakes on the Nankai Trough subduction zone [from Ando, 1975]. Refer to Figures 8 and $9 a$ for location key. 
PATCHES: NANKAI TROUGH

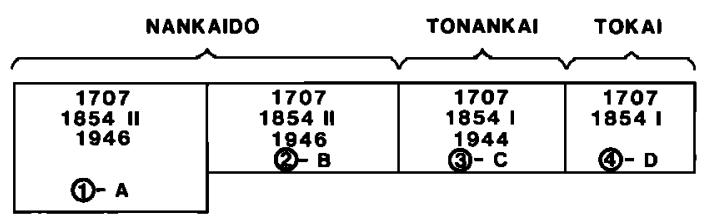

(a)

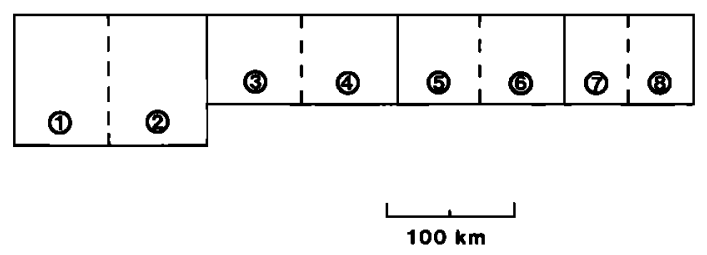

(b)

Fig. 9. Patch model for the Nankai Trough earthquakes. (a) A model adapted from that given by Ando [1975] and for which the geometric parameters are given in Table 5. Patches 1 and 2 (Ando's A and B) broke in the 1707, $1854 \mathrm{II}$, and 1946 events; patch 3 (Ando's fault plane C) broke in the $1707,1854 \mathrm{I}$, and 1944 events; and patch 4 (Ando's fault plane D) broke in the 1707 and 1854 I events. (b) An alternate model whose geometric parameters are the same as before but with the fault planes A-D divided in half to give eight patches.

the constant stress changes for each of the four patches as before. The moment of the 1707 event can then be calculated by making the totally simultaneous slip assumption. The result is given in Table $6 a$ : Clearly, although the calculated slip values for the 1707 event are larger than the values for slip on each patch singly, they are not large enough to match the 1707 values in Table 4. A possible way out of this difficulty is to hypothesize that each of the fault planes (A-D) in the Ando [1975] model (Figure 9a) is itself made up of two or more patches. For example, Figure $9 b$ shows an alternate model in which each of Ando's fault planes have been divided into two patches (designated 1-8). Again, we make the assumption that the 1707 event represents the simultaneous slip of all eight patches and that the other four events represent completely sequential slip (and are therefore minimum, or problem II, events). The static stress changes for all eight patches are then determined from the later four events, and the eight slip values for the 1707 event are then calculated as before. The results

TABLE 5. Parameters: Nankai Trough Earthquakes

\begin{tabular}{lcccc}
\hline & \multicolumn{4}{c}{ Patches } \\
\cline { 2 - 5 } & $1(\mathrm{~A})$ & $2(\mathrm{~B})$ & $3(\mathrm{C})$ & $4(\mathrm{D})$ \\
\hline Length, km & 150 & 150 & 130 & 100 \\
Width, km & 100 & 70 & 70 & 70 \\
Dip angle & $25^{\circ}$ & $25^{\circ}$ & $25^{\circ}$ & $25^{\circ}$ \\
Slip angle* & $68^{\circ}$ & $68^{\circ}$ & $68^{\circ}$ & $68^{\circ}$ \\
\hline
\end{tabular}

Faulting parameters for Nankai Trough earthquakes. Refer to Figure $9 a$ for details.

${ }^{*}$ Pure thrust $=90^{\circ}$.
TABLE 6a. Summary of Calculated Results for the Nankai Trough Earthquake Model: A Minor Variation on Ando's Original Model

\begin{tabular}{cccc}
\hline Patch & $\begin{array}{c}\text { Patch Stress Drop, } \\
\mathrm{MPa}\end{array}$ & $\begin{array}{c}\text { Single Patch Slip, } \\
\mathrm{m}\end{array}$ & $\begin{array}{c}1707 \text { Slip, } \\
\mathrm{m}\end{array}$ \\
\hline 1 & -4.130 & 6.0 & 6.9 \\
2 & -3.457 & 4.0 & 6.1 \\
3 & -3.620 & 4.0 & 6.0 \\
4 & -4.016 & 4.0 & 5.1 \\
\hline
\end{tabular}

Patch stress changes were calculated by finding the stress change for each patch, slipping alone, that gives the slip value shown in column 3 [from Ando, 1975]. See Figure 9a.

are given in Table $6 b$, and as can be seen, are closer to the values given by Ando [1975] (see Table 4). The reason that smaller patches yield larger simultaneous slip is that smaller patches need larger stress changes for the same size offset. So, to match the moments for the four later events, which have been assumed to be minimum size events, the eight patches need larger stress changes. When these same patches are then allowed to slip simultaneously, the offset is therefore larger than for the four original patches (compare Tables $6 a$ and $6 b$; note that the slip in the four later events is the same on the four original fault planes).

\section{Discussion AND Conclusions}

The fundamental concern of this paper has been the role of physical interactions between various faults and various segments of a particular fault plane. In order to quantify these effects we have developed a computational mechanism which we call the interaction matrix. This matrix is derived from the energy change for a general system of interacting cracks or faults; it relates the area-averaged stress state on a particular fault to the area-averaged offset state on all the other faults in the system. Because the interaction matrix is derived from the energy change, the area-averaged stress and slip state on a particular fault turn out to be energy consistent, an important constraint. Using these techniques, we demonstrated that earthquakes off the Colombia-Ecuador coast and in the Nankai Trough near Japan reveal temporal variations in seismic moment which are strong evidence for the importance of the interaction effects.

A question which we have not dealt with in any detail is how the various patches "communicate" their respective stress

TABLE 6b. Summary of Calculated Results for the Nankai Trough Earthquake Model: A Modification of Table $6 a$ in Which Each of the Four Fault Planes A-D is Divided Into Two Patches With Half the Fault Length

\begin{tabular}{cccc}
\hline Patch & $\begin{array}{c}\text { Patch Stress Drop, } \\
\text { MPa }\end{array}$ & $\begin{array}{c}\text { Single Patch Slip, } \\
\text { m }\end{array}$ & $\begin{array}{c}\text { 1707 Slip, } \\
\text { m }\end{array}$ \\
\hline 1 & -6.107 & 6.0 & 10.1 \\
2 & -6.107 & 6.0 & 10.1 \\
3 & -4.641 & 4.0 & 8.4 \\
4 & -4.641 & 4.0 & 8.4 \\
5 & -5.049 & 4.0 & 8.4 \\
6 & -5.049 & 4.0 & 8.4 \\
7 & -6.016 & 4.0 & 7.6 \\
8 & -6.016 & 4.0 & 7.6 \\
\hline
\end{tabular}

Patch stress changes were calculated by finding the stress change for each patch, slipping alone, that gives the slip value shown in column 3 [from Ando, 1975]. 
levels to each other during a slip event, a necessary prerequisite for the patch model to work. Such communication is a requirement for the various patches to "adjust" the amount of slip needed to satisfy the constancy of static stress drop. This implies that the source process time must be longer than the total time needed for seismic waves to propagate across the zone of influence of a particular patch for that patch to "know" what its neighbors are doing. Support for smaller patches, with dimensions of the order of the crustal thickness, comes from the evident disparity in moment between events on the same fault planes (see the discussion at the end of section 5). Moreover, a longer source process time is consistent with the idea that "slow earthquakes" [Kanamori and Stewart, 1979; Gladwin and Johnston, 1986] are an important part of the rupture process. Slow earthquakes can serve to extend the source process time, allowing the various patches to respond to each other's stress level.

An alternative view of this process is that following the progression of the rupture front outward from the hypocenter, there follows at some later time a "healing front" which spreads outward from some "healing center" at some "healing velocity," eventually covering the entire fault plane as well. This kind of picture would allow various patches on the fault plane to be sliding or healing at the same time during the event. Our simple model, in which the stress interaction coefficients are calculated from time-independent Green's functions, is then an aggregate representation of this entire process.

Using our patch model, we demonstrated that major earthquakes, which occur on a number of patches simultaneously, can produce significantly greater slip on the patches than do smaller events involving fewer patches. Again, these differences are due primarily to the effects of fault-fault interactions and therefore also on the details of slip initiation and healing on the various patches. In contrast to some previous authors who demand that stress following an earthquake be uniform over a fault plane our model allows stress to be spatially inhomogeneous both before and after the event. We illustrated these ideas by analytic and numerical calculations, and applied them to explaining the Colombia-Ecuador and Nankai Trough earthquake sequences, for which reasonable quantitative agreement was obtained.

Acknowledgments. The authors would like to acknowledge stimulating discussions with John Rudnicki and with Peter Malin. This research was supported by a grant from the National Aeronautics and Space Administration, Crustal Dynamics Program, to Sandia National Laboratories and by the U.S. Department of Energy under contract DE-AC04-76DP00789 to Sandia National Laboratories.

\section{REFERENCES}

Aki, K., Characterization of barriers on an earthquake fault, J. Geophys. Res., 84, 6140-6148, 1979.

Ando, M., Source mechanisms and tectonic significance of historical earthquakes along the Nankai Trough, Japan, Tectonophysics, 27, $119-140,1975$.

Beck, S. L., and L. J. Ruff, The rupture process of the great 1979 Colombia earthquake: Evidence for the asperity model, $J$. Geophys. Res., 89, 9281-9291, 1984.
Das, S., and K. Aki, Fault planes with barriers: A versatile earthquake model, J. Geophys. Res., 82, 5658-5670, 1977.

Dieterich, J. H., Constitutive properties of faults with simulated gouge, in Mechanical Behavior of Crystal Rocks, Geophys. Monogr. Ser., vol. 24, edited by N. L. Carter, M. Friedman, J. M. Logan, and D. W. Stearns, pp. 103-120, AGU, Washington, D. C., 1981.

Gladwin, M. T., and M. J. S. Johnston, Coseismic moment and total moment of the April 24, Morgan Hill and the January 26, 1986, Quiensabe earthquake (abstract), Eos. Trans. $A G U, 67,308,1986$.

Jaeger, J. C., and N. G. W. Cook, Fundamentals of Rock Mechanics, Chapman and Hall, London, 1969.

Kanamori, H., Tectonic implications of the 1944 Tonankai and the 1946 Nankaido earthquakes, Phys. Earth Planet. Inter., 5, 129-139, 1972.

Kanamori, H., The nature of seismicity patterns before large earthquakes, in Earthquake Prediction: An International Review, Maurice Ewing Ser., vol. 4, edited by D. W. Simpson and P. G. Richards, pp. 1-19, AGU, Washington, D. C., 1981.

Kanamori, H., and $\mathbf{K}$. C. McNally, Variable rupture mode of the subduction zone along the Ecuador-Colombia coast, Bull. Seismol. Soc. Am., 72, 1241-1253, 1982.

Kanamori, H., and G. S. Stewart, A slow earthquake, Phys. Earth Planet Inter., 18, 167-175, 1979.

Kelleher, J., Rupture zones of large South American earthquakes and some predictions, J. Geophys. Res., 77, 2087-2103, 1972.

Lay, T., H. Kanamori, and L. Ruff, The asperity model and the nature of large subduction zone earthquakes, Earthquake Predict. Res., I, 3-71, 1982.

Madariaga R., On the relation between seismic moment and stress drop in the presence of stress and strength heterogeneity, $J$. Geophys. Res., 84, 2243-2250, 1979.

Mansinha, L., and D. E. Smylie, The displacement fields of inclined faults, Bull. Selsmol. Soc. Am., 61, 1433-1440, 1971.

Maruyama, T., Statical elastic dislocations in an infinite and semiinfinite medium, Bull. Earthquake Res. Inst. Univ. Tokyo, 42, 289$368,1964$.

Rice, J. R., Constitutive relations for fault slip and earthquake instabilities, Pure Appl. Geophys., 121, 443-475, 1983.

Rice, J. R., and S. T. Tse, Dynamic motion of a single degree of freedom system following a rate and state dependent friction law, $J$. Geophys. Res., 91, 521-530, 1986.

Rudnicki, J. W., and H. Kanamori, Effects of fault interaction on moment, stress drop, and strain energy release, J. Geophys. Res., 86, 1785-1793, 1981.

Rudnicki, J. W., K. Hirashima, and J. D. Achenbach, Amplification of moment and strain energy release due to interaction between different size fault slip zones, J. Geophys. Res., 89, 5828-5834, 1984.

Ruina, A. L., Slip instability and state variable friction laws, J. Geophys. Res., 88, 10,359-10,370, 1983.

Rundle, J. B., H. Kanamori, and K. C. McNally, An inhomogeneous fault model for gaps, asperties, barriers, and seismicity migration, $J$. Geophys. Res., 89, 10,219-10,231, 1984. (Correction, J. Geophys. Res., $91,2218,1986$.

Sieh, K. E., Slip along the San Andreas fault associated with the great 1857 earthquake, Bull. Seismol. Soc. Am., 68, 1421-1448, 1978.

Stuart, W. D., Strain-softening instability model for the San Fernando earthquake, Science, 203, 907-910, 1979.

Stuart, W. D., Instability model for recurring large and great earthquakes in southern California, Pure Appl. Geophys., 122, 793-811, 1985.

H. Kanamori, Seismological Laboratory, California Institute of Technology, Pasadena, CA 91125.

J. B. Rundle, Division 1541, Sandia National Laboratories, Albuquerque, NM 87185 .

(Recelved April 21, 1986;

revised December 1, 1986;

accepted December 2,1986 .) 